\title{
3. Umwelt und Entwicklung
}

Catherine Schümperli Younossian and Xavier Tschumi Canosa

\section{(2) OpenEdition \\ 1 Journals}

Electronic version

URL: http://journals.openedition.org/sjep/841

DOI: $10.4000 /$ sjep.841

ISSN: 1663-9677

Publisher

Institut de hautes études internationales et du développement

\section{Printed version}

Date of publication: 1 avril 2001

Number of pages: $215-240$

ISSN: $1660-5926$

\section{Electronic reference}

Catherine Schümperli Younossian und Xavier Tschumi Canosa, « 3. Umwelt und Entwicklung », Schweizerisches Jahrbuch für Entwicklungspolitik [Online], 20 | 2001, Online erschienen am: 02 September 2012, abgerufen am 07 September 2020. URL : http://journals.openedition.org/sjep/841 ; DOI : https://doi.org/10.4000/sjep.841 


\section{UMWELT UND ENTWICKLUNG}

\subsection{NACHHALTIGE ENTWICKLUNG IN DER SCHWEIZ}

In der neuen Bundesverfassung wird die nachhaltige Entwicklung explizit erwähnt. Das Bundesamt für Raumentwicklung (ARE) im Departement für Umwelt, Verkehr, Energie und Kommunikation (UVEK) ist seit Juni 2000 in Betrieb. Das neue Amt hat namentlich den Auftrag, die Strategie des Bundesrates im Bereich nachhaltige Entwicklung umzusetzen. Der Rat für nachhaltige Entwicklung, den der Bundesrat 1998 eingesetzt hat, wurde Ende 2000 aufgelöst. Seine Arbeit wird vom Rat für Raumorganisation weiter geführt. Der Bundesrat hat, wie in seinen Zielen 2000 angekündigt, einen Zwischenbericht über den Stand der Umsetzung seiner Strategie der nachhaltigen Entwicklung abgelegt. Das UVEK definiert seine Strategie, für welche die nachhaltige Entwicklung als Leitfaden dient. Der interdepartementale Ausschuss für die Nachfolgearbeiten von Rio (IDA-Rio) bereitet sich mit dem «Projekt nachhaltige Entwicklung in der Schweiz» auf die Zehnjahreskonferenz «Rio+10» vor.

\section{$\square$ Die nachhaltige Entwicklung im institutionellen Rahmen des Bundes}

- Neue Bundesverfassung

Seit dem 1. Januar 2000 hat die Schweiz eine neue Bundesverfassung, in welcher die nachhaltige Entwicklung formell festgeschrieben ist. Das «Projekt nachhaltige Entwicklung in der Schweiz» beruft sich ausdrücklich darauf (s. weiter unten).

Die neue Bundesverfassung bezieht sich sowohl im Vorwort wie bei den Zielen, aber ebenso in einzelnen Artikeln zu den Aussenbeziehungen der Schweiz, zu Umwelt und Raumplanung, zum Verkehr, zur Energie, zur Landwirtschaft und zu den Finanzen auf die nachhaltige Entwicklung.

a Legislaturprogramm 1999-2003

Der Bundesrat arbeitet alle vier Jahre ein Legislaturprogramm aus, welches in Verbindung mit dem Finanzplan des Bundes die Ziele und Aktivitäten festlegt, die er in diesem Zeitraum umsetzen möchte. Seit der Konferenz von Rio 1992 widerspiegeln die Berichte, die der Bundesrat dem Parlament zu diesem Thema vorlegt, ausdrücklich den politischen Willen zur Berücksichtigung der nachhaltigen Entwicklung.

๖ Auflösung des Rats für nachhaltige Entwicklung

Der Rat für nachhaltige Entwicklung, der Anfang 1998 vom Bundesrat eingesetzt worden war, wurde im Mai 2000 mit Wirkung auf Ende des gleichen Jahres aufgelöst. Diese ausserparlamentarische (dem UVEK angegliederte) vorberatende Kommission war aus dreizehn unabhängigen Mitgliedern der Bundesbehörden zusammengesetzt und hatte den spezifischen Auftrag, die Bundesbehörden mittels Vorschlägen und durch eine Evaluation von deren Politik auf die nachhaltige Entwicklung hin auszurichten. 
Mit der Thematik der nachhaltigen Entwicklung wurde seither eine andere (dem eidgenössischen Volkswirtschaftsdepartement angegliederte) ausserparlamentarische vorberatende Kommission beauftragt, der Rat für Raumorganisation (ROR), der im Juni 1997 geschaffen wurde. Die Verordnung1, welche den Auftrag des Rats festschreibt, wurde deshalb am 18. Oktober 2000 abgeändert: der ROR erhielt als zusätzliche Aufgabe «die Vertiefung von Fragen der nachhaltigen Entwicklung und die Förderung von Synergien mit der Raumordnung ${ }^{2}$. Es ist vorgesehen, dass der Bundesrat Vakanzen und Rücktritte nutzt, "um die Kompetenzen des Rates im Bereich der nachhaltigen Entwicklung zu verstärken $»^{3}$.

- Neues Bundesamt für Raumentwicklung

Die Auflösung des Rates für nachhaltige Entwicklung geht zum Teil auf Schaffung des Bundesamtes für Raumentwicklung (ARE) im UVEK zurück. Das ARE betreut bereits den ROR (s. oben), und nach Ansicht des Bundesrates konnte das relativ kleine Amt nicht auch noch einen Rat für nachhaltige Entwicklung «mit einem verwandten Pflichtenheft» betreuen ${ }^{4}$. Das Bundesamt für Raumplanung im eidgenössischen Justiz- und Polizeidepartement (EJPD) wurde mit Beschluss des Bundesrates unter dem Namen ARE dem UVEK angegliedert: "Die Politik des Bundes in der Raum- und Verkehrsentwicklung, der nachhaltigen Entwicklung und der Alpenkonvention wird ab dem 1. Juni vom Bundesamt für Raumentwicklung vorbereitet und umgesetzt. $»^{5}$

\section{$\square$ Bilanz der Strategie des Bundesrats im Bereich nachhaltige Entwicklung}

1997 hiess der Bundesrat die Strategie für eine «nachhaltige Entwicklung in der Schweiz» gut, die aus acht verschiedenen Aktionsfeldern mit elf Massnahmen besteht. Drei Jahre später, im Dezember 2000, zieht er in einem Zwischenbericht $^{6}$, der vom interdepartementalen Ausschuss für die Nachfolgearbeiten von Rio (IDA-Rio) erstellt wurde, Bilanz über die Umsetzung dieser Massnahmen.

@ JSDW 1998, Kapitel 4.1, S. 192-195.

In diesem Bericht werden alle Fortschritte aufgezeigt, welche seit der Festlegung der Strategie erreicht wurden, es wird aber auch festgehalten, was noch zu erreichen ist. Die meisten Massnahmen sind erst teilweise umgesetzt, zwei dagegen sind bereits vollständig realisiert: die Erarbeitung eines gemeinsamen Konzepts der nachhaltigen Entwicklung im Bereich internationale Politik (Aktionsfeld internationales Engagement) und die Schaffung eines Rats für nachhaltige Entwicklung (Aktionsbereich Umsetzung und Erfolgskontrolle). Die Massnahme, welche die Besteuerung der Energie und die Entlastung des Faktors Arbeitskraft

1. Verordnung vom 22.10.1997 über die raumpolitische Koordination der Bundesaufgaben (709.17), 4 S.

2. Verordnung vom 22.10.1997 über die raumpolitische Koordination der Bundesaufgaben, Art 5, Abs.2 Bst. d.

3. Antwort des Bundesrates auf die Interpellation 00.3554 (Eymann Christoph), 11.12.2000.

4. Ibid

5. UVEK, Bundesamt für Raumentwicklung, Pressemitteilung vom 17.5.2000

6. UVEK «Die nachhaltige Entwicklung in der Schweiz: Bilanz nach drei Jahren», Pressemitteilung vom 11.12.2000. Über einen Internetlink am Ende der Mitteilung kann der Zwischenbericht des Bundesrates gelesen oder heruntergeladen werden: www.buwal.ch/presse/2000/d0012111.htm. 
zum Ziel hat (Aktionsfeld Steuerreform nach ökologischen Kriterien), konnte nicht umgesetzt werden, da am 24. September 2000 sowohl die Förderabgabe wie die Energielenkungsabgabe für die Umwelt an der Urne abgelehnt wurden. Dass die Umsetzung der Massnahmen erst teilweise geschehen ist, ist erklärbar durch die weit gefassten Forderungen, die nur langsam erfüllt werden können. Die Umsetzung der bundesrätlichen Strategie der nachhaltigen Entwicklung ist ein Prozess, der ausdrücklich auf Dauer angelegt ist.

\section{$\square$ Die neue Strategie des UVEK}

Das UVEK geht mit einer neuen Strategie ${ }^{7}$, die auf nachhaltige Entwicklung ausgerichtet ist, in die Legislaturperiode 1999-2003. Diese Strategie ist ein Führungsinstrument für die gesamte Arbeit des Departements mit seinen vier Aktivitätsbereichen Umwelt, Verkehr, Energie und Kommunikation. Sie legt die Leitlinien des UVEK fest, sowohl im internen wie im externen Bereich (Einsichtsrecht der Öffentlichkeit in die Aktivitäten des Departements).

In jedem dieser Bereiche bauen die Ziele auf drei Dimensionen der nachhaltigen Entwicklung auf, nämlich der ökologischen Durchführbarkeit, der wirtschaftlichen Effizienz und der sozialen Gerechtigkeit.

Die Strategie beschreibt auch im Detail die Prinzipien, von welchen die entsprechenden Aktivitäten des UVEK geleitet werden, ebenso die wichtigen Massnahmen, die ergriffen werden müssen.

\section{$\square$ «Projekt nachhaltige Entwicklung in der Schweiz»}

Für das Projekt nachhaltige Entwicklung in der Schweiz ${ }^{8}$, das 1999 lanciert wurde, ist der interdepartementale Ausschuss für die Folgearbeiten von Rio (IDA-Rio) zuständig. Der Ausschuss erhielt 1993 den Auftrag, die Umsetzung der Agenda 21 in der Schweiz zu koordinieren. Finanziert wird das Projekt von der DEZA und vom BUWAL, die operative Leitung liegt bei der DEZA.

Das Projekt begann Anfang 2000 und wird bis zur Konferenz «Rio+10» weitergeführt. Diese wird Bilanz ziehen über die seit dem Erdgipfel von 1992 in Rio erreichten Fortschritte auf dem Weg in Richtung nachhaltige Entwicklung.

Die Arbeiten sind durch mehrere Etappen gekennzeichnet, namentlich durch den Zwischenbericht des Bundesrates über seine 1997 festgelegte Strategie der nachhaltigen Entwicklung (s. weiter oben). Der Bericht über das Projekt soll zwischen 2001 und 2002 in einer allen zugänglichen Art veröffentlicht werden.

Beim Projekt geht es erstens um einen normativen Bezugsrahmen, der auf einer ethischen Interpretation der nachhaltigen Entwicklung aufbaut. Als Zweites wird die Situation des Bundes in Bezug auf die nachhaltige Entwicklung in fünf Bereichen berücksichtigt (Gesellschaft, Wirtschaft, Umwelt, Aussenpolitik und politische Institutionen), in denen möglichst kohärente Politiken verfolgt werden müssen, und wo zur Zeit noch einige Zielkonflikte bestehen.

Das Projekt zeigt, hauptsächlich in acht Bereichen Perspektiven für die nachhaltige Entwicklung auf (Humanressourcen, Solidarität, der Platz der Schweiz in

7. Internet: www.uvek.admin.ch/doku/presse/2000/d/00010603.pdf.

8. Die 4-seitige Publikation mit dem Titel «Projekt nachhaltige Entwicklung in der Schweiz. Standortbestimmung und Perspektiven » ist gratis erhältlich bei der DEZA, Sektion Politik und Forschung (im Internet unter: www.deza.amin.ch) 
der Welt, die Fähigkeit der Wirtschaft, die Zukunft anzupacken, Konsumgewohnheiten, Gesundheit, natürliche Lebensgrundlagen und institutioneller Rahmen).

Mit dem Projekt sollen Aktionsfelder identifiziert werden, welche die in der Strategie des Bundesrates enthaltenen Felder ergänzen, wie zum Beispiel die Raumplanung.

\section{INTERNET-ADRESSEN}

UVEK: www.uvek.admin.ch

BUWAL: www.buwal.admin.ch

DEZA: www.deza.admin.ch

Bundsamt für Raumentwicklung: www.raumentwicklung.admin.ch (provisorische Website)

Rio+10: www.un.org/rio+10/default.htm

\subsection{UMSETZUNG DER RAHMENKONVENTION ÜBER KLIMAÄNDERUNGEN UND DES PROTOKOLLS VON KYOTO}

Die 6. Konferenz der Vertragsparteien (COP6) der Rahmenvereinbarung über Klimaänderungen (Uno-Klimakonvention) fand vom 13. bis 25. November 2000 in Den Haag statt. Da in den Hauptpunkten der Verhandlungen keine Einigung zustande kam, wurde die Konferenz vertagt. Die Parteien wurden aufgefordert, bis zur Wiederaufnahme eine Verständigungsgrundlage zu finden, damit weder die Umsetzung der Konvention noch der Ratifizierungsprozess des Protokolls von Kyoto verzögert werden. An dieser Konferenz trat die Schweiz namentlich dafür ein, dass die Industriestaaten zuerst ihren eigenen Ausstoss an Treibhausgasen abbauen müssen. Dafür setzt sich auch die Europäische Union ein, dies läuft aber der Position der USA und der Länder der Umbrella group (Vereinigte Staaten, Kanada, Russland, Ukraine, Australien, Neuseeland, Norwegen, Island und Japan) zuwider.

Die Frage der durch den Luftverkehr verursachten Treibhausgase war Thema einer Studie des schweizerischen Nationalfonds für wissenschaftliche Forschung.

Im Rahmen seiner Energiepolitik hat die Schweiz ein $\mathrm{CO}_{2}$-Gesetz eingeführt (seit dem 1. Mai 2000 in Kraft). Sie zieht ausserdem die Lehren aus dem dreifachen Nein bei der Volksabstimmung vom 24. September 2000. Die Schweiz zieht ferner Bilanz über ihr Energieprogramm 2000 und bereitet die Umsetzung des Nachfolgeprogramms «EnergieSchweiz» vor, das im Januar 2001 anlief.

\section{$\square$ Die 6. Vertragsparteienkonferenz, Den Haag (13.-25. November 2000)}

Die Delegationen aus den 177 Ländern, welche Vertragsparteien der Rahmenvereinbarung der Vereinten Nationen über Klimaveränderungen (Uno-Klimakonvention) sind, wozu noch Delegationen aus vier Beobachterstaaten kamen, traten in Den Haag zum sechsten Mal zusammen, mit dem Ziel, die Modalitäten der Umsetzung des Protokolls von Kyoto festzulegen und jene der Konvention weiter zu verfolgen. Das Protokoll verlangt einzig von den Industriestaaten (38 Länder, darunter Russland, welche im Anhang I genannt sind), dass sie ab 2008 bis 2012 die Gesamtemission ihrer Treibhausgase um mindestens 5\% gegenüber 
1990 senken (für jedes Land gilt ein anderer Prozentsatz). Das Protokoll wird erst in Kraft treten und rechtlich bindend sein, wenn es von 55 Parteien der in Anhang I genannten Länder, auf die 55\% der Gesamtemissionen an Treibhausgasen fallen, ratifiziert ist.

凹 JSDW 2000, S.182-190.

Der Erfolg der COP6 gilt als wesentliche Vorbedingung, damit der Ratifizierungsprozess des Protokolls eingeleitet werden kann: bei den grundlegenden Themen, die dabei verhandelt werden, geht es vor allem um Flexibilitätsmechanismen, welche vom Protokoll zugelassen sind, sowie um die Fragen der Supplementarität, der Festlegung für natürliche Kohlenstoffspeicherung (LULUCF für Land-Use, Land-Use Change and Forestry), um die Respektierung der Verpflichtungen (System der gegenseitigen Abstimmung) und um Finanzierungsfragen (insbesondere zur Milderung negativer Auswirkungen, für den Technologietransfer und die Anpassungsmassnahmen).

Unter all den Gruppen von Ländern, welche sich um diese Hauptherausforderungen gruppiert haben und sich in den Verhandlungen für bestimmte Interessen einsetzten, gehörten jene der Europäischen Union, der sich im Allgemeinen die übrigen Länder Europas anschliessen, und jene der USA, Kanadas, Russlands, der Ukraine, Australiens, Neuseelands, Norwegens, Islands und Japans (eine flexible Allianz, die sich Umbrella group nennt) zu den beiden Hauptkräften unter den Industrienationen. Die Gruppe von Entwicklungsländern, welcher sich 133 Länder anschlossen, und zu der auch China gezählt werden muss (G77/China), ist die drittstärkste Kraft und vertritt eine von den beiden ersten Gruppen abweichende Politik. Die Schweiz hat eine informelle Verhandlungsgruppe mit Namen «Gruppe für die Umweltintegrität» gebildet (s. unten, Position der Schweizer Delegation).

In der ersten Woche der Konferenz sollten die seit den Sitzungen der Subsidiärorgane $^{9}$ der Uno-Klimakonvention in Bonn (SB-12, 12-16.6.2000) und Lyon (SB-13, 11.-15.11.2000) noch hängigen technischen und wissenschaftlichen Fragen so weit wie möglich gklärt werden. Diese Sitzungen gingen zu Ende, ohne dass die Ländergruppen einen Konsens in Bezug auf die Verhandlungstexte fanden, zahlreiche Paragraphen wurden in Klammern gesetzt. Trotz intensiver Verhandlungen während der ersten Woche in Den Haag (welche eigentlich der zweite Teil der SB-13 war) waren die Teilnehmer vor Beginn der zweiten Konferenzwoche auf Ministerebene nach wie vor sehr gespalten.

Diese Verhandlungen auf Ministerebene führten nicht zu einer Annäherung der Standpunkte zwischen der Europäischen Union und den USA (als Anführer ihrer jeweiligen Gruppe). «Zwei Philosophien stehen sich gegenüber: der amerikanische Merkantilismus und der europäische Interventionismus ${ }^{10}$. Die Vereinigten Staaten streben zur Berechnung der Reduktion der Treibhausgasemissionen eine möglichst weitgehende Anwendung der flexiblen Mechanismen und die maximale Berücksichtigung der Kohlenstoffspeicherung an, während die Europäische

9. Der Uno-Klimakonvention gehören zwei hauptsächliche Subsidiärorgane an, das Subsidiärorgan für wissenschaftlichen und technologischen Beratung (Subsidiary Body for Scientific and Technological Advice - SBSTA) und das Subsidiärorgan für die Umsetzung des Rahmenübereinkommens (Subsidiary Body for Implementation - SBI).

10. L'AGEFI, «Cent quatre-vingts pays au chevet de la planète», 15.11.2000. 
Union für eine beschränkte Anwendung der flexiblen Mechanismen einstehen und die gesamte Verrechnung der Kohlenstoffspeicherung ablehnen. Und was das System der gegenseitigen Abstimmung angeht, sprachen sich die meisten Parteien für eine Regelung mit vielen Sanktionen aus (aber nur, wenn das erleichternde Verfahren, das den Ländern bei der Erreichung ihrer Abbauziele bei den Treibhausgasen helfen soll, keine Wirkung zeigt). Allerdings stiessen auch in diesem Zusammenhang technische und juristische Fragen auf unterschiedliche Standpunkte.

Angesichts dieser Blockierung, und weil der Abschluss der Konferenz für den nächsten Tag vorgesehen war, unterbreitete der Konferenzpräsident ${ }^{11}$ den Unterhändlern eine Note, die einen Konsens herbeiführen sollte. Die Minister fanden den Konsens weder an diesem letzten Konferenztag noch in der darauf folgenden Nacht. Am Nachmittag des 25. November, also mit einem Tag Verspätung auf die offiziellen Tagesordnung, vertagte der Präsident die Konferenz. In der Plenarsitzung wurde darauf hingewiesen, dass trotz allem bei allen Fragen zu dem in Buenos Aires festgelegten Aktionsplan einige Fortschritte erzielt worden waren. Die Punkte, wo die Verhandlungen noch blockiert sind und bei denen eine politische Einigung erreicht werden muss, wurden an der COP6 klar festgehalten.

凹 JSDW 2000, S. 182-190.

Der von der Konferenz verabschiedete Beschluss betrifft fünf Punkte ${ }^{12}$ :

- Die Note des Präsidenten vom 23. November 2000 dient den Parteien bei ihren Verhandlungsarbeiten als politischer Wegweiser.

- Die Parteien wurden aufgefordert, sich bis zum 15. Januar 2001 zur Note des Konferenzpräsidenten und zu anderen Dokumenten zu äussern.

- Die Konferenz wurde vertagt und der Präsident muss prüfen, ob es zweckdienlich ist, die Arbeiten der Konferenz im Mai/Juni 2001 (vermutlich in Bonn anlässlich der Sitzung SB-14) wieder aufzunehmen, um die Verhandlungen zum Ende zu führen und ausgeglichene Beschlüsse zu verabschieden, welche den Zielen des Aktionsplans von Buenos Aires gerecht werden.

- Der Präsident muss den Parteien Vorschläge zu den Verhandlungstexten unterbreiten und auf transparente Weise ihre Meinung einholen, bevor die Arbeiten der Konferenz wieder aufgenommen werden.

- Die Parteien müssen die politischen Beratungen intensivieren und eine Verständigungsgrundlage finden, um den Erfolg der Verhandlungen gemäss dem Aktionsplan von Buenos Aires zu gewährleisten.

Am 6. und 7. Dezember nahmen die Europäische Union (Frankreich, Grossbritannien, Schweden, Deutschland) und die USA (mit den Vertretern Kanadas, Japans und Australiens) die Gespräche in Ottawa wieder auf, doch wurde keine Mitteilung dazu veröffentlicht (privates Treffen).

11. Der holländische Umweltminister Jan Pronk.

12. Der Beschluss 1/CP.6, gefolgt von der Note des Präsidenten, ist auf Englisch abrufbar unter der Internetadresse der Klimakonvention: www.unfccc.int/resource/docs/cop6/dec1-cp6.pdf. 


\section{$\square$ Position der Schweizer Delegation}

Die Schweizer Delegation in Den Haag wurde von Philippe Roch, Direktor des Bundesamtes für Umwelt, Wald und Landschaft (BUWAL) geleitet. Roch wurde von einer Verhandlungsdelegation unter Leitung von Botschafter Beat Nobs (BUWAL) begleitet. Dieser Delegation gehörten Mitglieder von drei eidgenössischen Departementen an (BUWAL, EDA und EVD), Vertreter und Vertreterinnen aus Wirtschaft und Wissenschaft sowie von Umwelt- und Hilfsorganisationen.

An der Sitzung SB-13 in Lyon hatte die Schweiz die Initiative ergriffen und eine eigentliche Verhandlungsgruppe gebildet und geleitet, die «Gruppe für Umweltintegrität» (Environmental Integrity Group, EIG), der auch Südkorea, Mexiko, Liechtenstein und Monaco angehören.

Eines der umstrittenen Themen der COP6, für das sich die Schweizer Delegation besonders einsetzte, war die Frage der Kohlenstoffspeicherung. Sie räumte zwar ein, dass die Speicherung zum Abbau des Treibhauseffektes beitragen kann, vertrat aber die Ansicht, dass die Verrechnung mit dieser Speicherung nur zugelassen sein sollte,

口 wenn sie ökologisch im weitesten Sinn sinnvoll ist,

๑ wenn sie keine absurden Anreize schafft ${ }^{13}$ und

- wenn sie in nachhaltiger Weise zur Reduktion der Treibhausgase in der Atmosphäre beiträgt.

Die Position der EIG wurde offenbar in der ersten Verhandlungswoche und dann auch auf Ministerebene verteidigt, aber die Konferenz wurde vertagt, bevor zur Frage der Kohlenstoffspeicherung ein Beschluss gefasst wurde.

Parallel zu den Arbeiten der COP6 hat die Schweiz zwei Anlässe vorbereitet:

口 Präsentation der Aktivitäten des Programms National Strategic Studies der Weltbank, welche sie mitfinanziert. Ziel dieses Programms ist es, das Potenzial und die Bedürfnisse der Transitions- und Entwicklungsländer für die Umsetzung der flexiblen Mechanismen des Protokolls von Kyoto zu analysieren (Joint Implementation in den Transitionsländern und Clean Development Mechanism in den Entwicklungsländern).

- Präsentation des Resultats einer vom BUWAL in Auftrag gegebenen Studie über die Erhaltung der Artenvielfalt bei Anwendung der Kohlenstoffspeicherung (mit dem Ziel, Kohärenz und die Unversehrtheit der Umwelt zu gewährleisten). Diese Studie wird voraussichtlich zu Beginn des Jahres 2001 veröffentlicht.

\section{$\square$ Die Frage der durch den internationalen Verkehr verursachten Treibhausgasemissionen}

In der Klimakonvention werden die vom Luft- und Seeverkehr verursachten Treibhausgase nicht speziell erwähnt, obwohl allgemein anerkannt ist, dass die Frage im Prinzip auf alle Treibhausgasemissionen anwendbar ist.

13. Der Treibhauseffekt führt zu einer klimatischen Erwärmung und einer allgemeinen Zunahme der Feuchtigkeit, welche das Vegetationswachstum und damit die Bedeutung der Kohlenstoffspeicherung fördert. Dabei geht es um einen Windfall, der nach Ansicht der Schweizer Delegation nicht verrechnet werden darf, denn das Ziel besteht darin, den Treibhauseffekt zu vermindern. 
Im Protokoll von Kyoto dagegen werden diese Treibhausgasemissionen erwähnt, und die im Anhang I genannten Länder werden aufgefordert, die Emissionen zu begrenzen und sie über zwei auf diesen Bereich spezialisierte UNO-Organisationen, die Internationale Zivilluftfahrt-Organisation (ICAO) und die Internationale Schifffahrts-Organisation (IMO) zu reduzieren.

Jedes Land quantifiziert die vom eigenen und vom internationalen Luft- oder Seeverkehr verursachten Emissionen. Die im Inland verursachten Emissionen werden ins nationale Inventar jedes Landes aufgenommen, jene des internationalen Verkehrs werden aber nur aufgrund des in jedem Land verkauften Treibstoffes berechnet, ohne dass sie in irgendein Inventar aufgenommen werden. Bei der Anrechnung der durch den internationalen Verkehr verursachten Treibhausgasemissionen stellen sich sehr komplexe methodologische Fragen. Die SBSTA hat zwar mehrere Optionen vorgeschlagen, um darauf einzugehen, doch wurde von den Parteien noch kein Beschluss gefasst (die Diskussionen darüber wurden 1996 abgebrochen). Die durch den internationalen Luft- und Seeverkehr verursachten Treibhausgasemissionen können also nicht verrechnet werden, sie machen aber laut Angaben der intergouvernementalen Expertengruppe über die Klimaentwicklung (IPCC) nur 3\% des weltweiten Treibhausgasausstosses aus. Zum Vergleich: der vom Strassenverkehr verursachte Treibhausgassausstoss beläuft sich auf $31 \%$ der gesamten weltweiten Treibhausgasemissionen.

Der Gütertransport und die internationalen (Luft- und See-) Transporte ihrerseits verursachen ein grosse Menge an Treibhausgasen (zum Beispiel wird geschätzt, dass für jede von Neuseeland nach Europa transportierte Kiwifrucht fünf Mal deren Gewicht an Treibhausgasen in die Atmosphäre ausgestossen wird ${ }^{14}$ ). Ausserdem gehen Prognosen davon aus, dass der internationale Gütertransport ab 2008 bis 2012 um $70 \%$ zunehmen wird ${ }^{15}$.

Im Zusammenhang mit dem Reiseverkehr zeigt eine Studie ${ }^{16}$ des Schweizerischen Nationalfonds für wissenschaftliche Forschung auf, dass in zwanzig Jahren der Luftverkehr für rund einen Drittel des $\mathrm{CO}_{2}$-Ausstosses pro Kopf der in der Schweiz lebenden Bevölkerung und für $40 \%$ der gesamten zurückgelegten Kilometer verantwortlich sein wird ${ }^{17}$. Aus der gleichen Studie geht hervor, dass der Luftverkehr (Fracht, Reise- und Charterverkehr) zur Zeit eine Tonne Treibhausgase pro Kopf der in der Schweiz lebenden Bevölkerung und Jahr produziert, und dass diese Emissionen in keiner nationalen Bilanz auftauchen. Die Studie kommt zum Schluss, dass mit der vorhersehbaren Zunahme des Luftverkehrs diese Entwicklung «den Zielsetzungen der internationalen und der schweizerischen Klimapolitik diametral entgegenläuft ${ }^{18}$.

Die Studie wurde am 22. September 2000 in Basel im Rahmen der SUN21 anlässlich der dritten Internationalen Wochen für eine nachhaltige Energiezukunft vorgestellt.

14. Courrier international, «Fret: le vice caché des négociations sur le réchauffement climatique», Nr. 528, 14-20.12.2000, S. 78.

15. Ibid.

16. Dossier NFP 41 «Verkehr und Umwelt», Luftverkehr - eine wachsende Herausforderung für die Umwelt, Fakten und Trends für die Schweiz, Band M25. EDMZ-Bestellnummer 801671.d (www.admin.ch/edmz).

17. SNF «Flugverkehr: der unterschätzte Klimasünder», Pressemitteilung vom 18.9.2000

18. Ibid. 
In ihrem Spezialbericht ${ }^{19}$ schreibt die intergouvernementale Expertengruppe über Klimaänderungen (IPCC), dass zwischen 1990 und 2015 mit einem jährlichen weltweiten Wachstum des Luftverkehrs um 5\% und für die gleiche Periode mit einem Wachstum des Treibstoffverbrauchs um 3\% zu rechnen ist (unter Berücksichtigung der gesteigerten Effizienz der Flugzeuge).

In der Strategie des Departements für Umwelt, Verkehr, Energie und Kommunikation (UVEK) heisst es: «Das UVEK setzt sich international, insbesondere in Europa, für die Aufhebung der Befreiung von Steuern und Abgaben auf Flugtreibstoffen ein ${ }^{20}$. Die Abgaben sollen vorab der Finanzierung von Massnahmen zum Schutz der Umwelt und zur Senkung des Treibstoffverbrauchs dienen.

\section{$\square$ Die Energiepolitik der Schweiz}

- Das Bundesgesetz über die Reduktion der $\mathrm{CO}_{2}$ Emissionen $\left(\mathrm{CO}_{2}-\mathrm{Gesetz}\right)$ ist in Kraft

Seit dem 1. Mai 2000 ist das $\mathrm{CO}_{2}$-Gesetz in $\mathrm{Kraft}^{21}$. Es legt als Ziel eine Verminderung der $\mathrm{CO}_{2}$-Emissionen bis zum Jahr 2010 um insgesamt 10\% gegenüber 1990 fest. Damit kann die Schweiz ihre in Kyoto eingegangene Verpflichtung erfüllen (-8\% für diesen Zeitraum), obwohl das Protokoll noch nicht in Kraft ist. Die Emissionen aus der Nutzung fossiler Energieträger (Erdöl, Kohle, Gas) sollten um 15\% reduziert werden, jene aus fossilen Brennstoffen (Benzin, Diesel, aber ohne Flugtreibstoffe für internationale Flüge) um $8 \%$.

」 Das Gesetz zählt zunächst auf Massnahmen, auf die sich Wirtschaft und Einzelne freiwillig einigen, um diese Ziele zu erreichen. Die vom Bund bereits ergriffenen oder die noch vorgesehenen Massnahmen wie die leistungsabhängige Schwerverkehrsabgabe (LSVA) oder das Energiegesetz, die am 1. Januar 1999 in Kraft getreten sind (s. auch Energieprogramm 2000, weiter unten), werden das Ihre dazu beitragen.

Subsidiär sieht das Gesetz die Einführung einer $\mathrm{CO}_{2}$-Abgabe vor, wenn die Ziele nicht mit den erwähnten Massnahmen erreicht werden können. Diese Abgabe kann nicht vor 2004 erhoben werden und darf nicht mehr als $210 \mathrm{Fr}$. pro Tonne $\mathrm{CO}_{2}$ kosten (was einen Liter Benzin um maximal 50 Rappen verteuern würde). Die Einnahmen aus dieser Abgabe würden der Bevölkerung (nach einem noch festzulegenden Verfahren) und den Unternehmen (über die AHV-Ausgleichskassen) zurückerstattet.

a Solarinitiative und Energieförderabgaben in der Volksabstimmung

Die drei dem Stimmvolk am 24. September 2000 vorgelegten Energievorlagen hätten die Fördermassnahmen zur Reduzierung der $\mathrm{CO}_{2}$-Emissionen ergänzen sollen. Die Solarinitiative verlangte, dass auf nicht erneuerbaren Energien eine Abgabe von 0,5 Rappen pro Kilowattstunde erhoben werde, um damit die Solarenergie und andere erneuerbare Energiearten zu fördern. Sie wurde in letzter Instanz von Volk und Ständen abgelehnt (68,1\% Nein).

19. IPCC, L'aviation et l'atmosphère planétaire.

20. Departementsstrategie UVEK, S. 15. (die Strategie kann auf der Website des UVEK abgerufen werden unter: www.uvek.admin.ch/doku/presse/2000/d/00010603.pdf).

21. Bundesgesetz über die Reduktion der $\mathrm{CO}_{2}$-Emissionen $\left(\mathrm{CO}_{2}\right.$-Gesetz) vom 18. Oktober 1999 (SR 641.71) 
Der Gegenvorschlag (Energielenkungsabgabe für die Umwelt von 0,3 Rappen pro Kilowattstunde auf nicht erneuerbaren Energien), wurde ebenfalls von Volk und Ständen abgelehnt, wenn auch knapper (53,4 \% Nein). Damit wird das Projekt der ökologischen Steuerreform (Lenkungsabgabe von 2 Rappen pro Kilowattstunde auf nicht erneuerbaren Energien als Kompensation für eine Reduktion der AHV-Beiträge) verzögert.

Die Schweizer Wirtschaft hatte sich im Allgemeinen stark gegen diese Abgaben eingesetzt. Sie zog inhaltlich das $\mathrm{CO}_{2}-\mathrm{Gesetz}$ vor (namentlich die Schweizer Wirtschaftsförderung titelte in einer ihrer Pressemitteilungen am 1. Mai 2000: lieber das $\mathrm{CO}_{2}$-Gesetz als neue Steuern). Bundesrat Moritz Leuenberger will allerdings «die Gegner [dieser Steuer] beim Wort nehmen»22, das heisst die im $\mathrm{CO}_{2}$-Gesetz vorgesehene Steuer einführen, wenn sich zeigt, dass die Ziele anders nicht erreicht werden können. Das Bundesamt für Energie (BFE) zweifelt kaum an der Einführung dieser Abgabe, zeigen doch die jüngsten Energieprognosen, dass die Ziele des $\mathrm{CO}_{2}$-Gesetzes nicht erreicht werden dürften. ${ }^{23}$

- Bilanz des Programms Energie 2000 und Folgeprogramm EnergieSchweiz

Das 1991 lancierte Programm Energie 2000 verfolgte zwei sich ergänzende Achsen, welche auf dem Verfassungsauftrag zur Energiepolitik basieren, nämlich die rationelle Nutzung der Energie und die zunehmende Nutzung erneuerbarer Energien.

Entlang dieser Achsen wurden die Ziele und Mechanismen des Programms festgelegt. ${ }^{24}$ Angesichts der spärlichen finanziellen Mittel, welche zur Verfügung standen, ist die Bilanz von Energie 2000 ermutigend, hat aber nicht alle Ziele vollumfänglich erreicht, insbesondere nicht jenes der Stabilisierung des Verbrauchs fossiler Energieträger und des $\mathrm{CO}_{2}$-Ausstosses auf dem Niveau von 1990 (im Jahr 2000 hatte der Verbrauch fossiler Energien in der Schweiz im Vergleich zu 1990 um 11\% zugenommen ${ }^{25}$ ). Das SBI der Klimakonvention führt ein Inventar der von den Parteien verursachten Treibhausgasemissionen, das aufgrund der nationalen Meldungen erstellt wird ${ }^{26}$. Laut diesem Inventar machte die Zunahme des Treibhausgasausstosses in der Schweiz zwischen 1990 und 1998 nur 1\% aus (blieb also praktisch stabil).

Dagegen hat der Verbrauch an erneuerbaren Energien zwischen 1990 und 1998 um 12,5\% zugenommen. Dies ist ein indirekter Erfolg des Programms Energie 2000, hatte doch dieses eine gesteigerte Produktion erneuerbarer Energien zum Ziel. Fast die gesamte Zunahme geht auf die Wasserkraft zurück, aber auch Holz- und Sonnenenergie haben laut der ersten Schweizer Statistik über erneuerbare Energien, welche im Januar 2000 erschienen ist ${ }^{27}$ und jedes Jahr aktualisiert werden soll, einen grossen Anteil daran.

22. Le Temps, «Il faut appliquer la loi sur le $\mathrm{CO}_{2} », 25.9 .2000$.

23. BFE, Die Gegner in die Pflicht nehmen, Newsletter Nr. 3

24. S. Jahresbericht 1999 des Programms, S. 6. Abrufbar auf der Website Energie 2000: www.e2000.ch

25. BEW, «Erfolgskontrolle in der Energiepolitik: Weitere Energie 2000-Ergebnisse veröffentlicht », Pressemitteilung vom 4.7.2000.

26. Das letzte Inventar der in Anhang I genannten Länder ist auf dem Internet auf Englisch abrufbar:

27. BFE, Schweizerische Statistik der erneuerbaren Energien, (EDMZ-Bestellnummer 805.520.2 d, www.admin.ch/edmz). 
Dank Energie 2000 konnten aber wichtige Erfahrungen gesammelt werden, von denen das Folgeprogramm EnergieSchweiz profitieren dürfte. Dieses wurde ohne Unterbruch zu Energie 2000 am 30. Januar 2001 lanciert. Mit Veranstaltungen in allen Landesteilen wurde die Bevölkerung über das Programm informiert, «das alle angeht» 28 .

Nach der Ablehnung der Energieförderabgaben in der Volksabstimmung vom 24. September 2000 erhält EnergieSchweiz nur 50 statt der vorgesehenen 450 Millionen Franken, welche die Förderabgabe ermöglicht hätte. Die Mittel für das neue Programm müssen deshalb nach unten korrigiert werden, und die Erreichung der Ziele wird schwieriger als vorgesehen. Zum Beispiel braucht es, um die $\mathrm{CO}_{2}$-Emissionen im Jahr 2010 gegenüber 1990 um $10 \%$ zu reduzieren, sicher zusätzliche Massnahmen, namentlich die $\mathrm{CO}_{2}$ Abgabe.

\section{QUELLEN}

L'Agefi, «Conférence de la dernière chance à La Haye pour passer à l'offensive contre le réchauffement climatique» (6.11.2000). «Le bon élève suisse exige des efforts des pays industrialisés» (10.11.2000). «Cent quatre-vingts pays au chevet de la planète» (15.11.2000).

Neue Zürcher Zeitung, «Dürftiger Konsens in der Klimapolitik. Kein Durchbruch beim Treffen in Lyon» (16-17.09.2000). «Ordnung im eigenen Land. Schweizer Positionsbezug vor der Klimakonferenz» (10.11.2000). «Plädoyer für echte Emissionsreduktionen. Philippe Roch vor der Haager Klimakonferenz» (22.11.2000).

IISD, Earth Negociations Bulletin, vol. 12, $\mathrm{n}^{\circ} 163,27.11 .2000$ (Summary of the Sixth Conference of the Parties to the Framework Convention on Climate Change: 13-25 November 2000). Abrufbar auf der Internetadresse: www.iisd.ca/climate/cop6/.

UVEK, «Weltklimakonferenz in den Haag (NL) vom 13. bis 24. November: Für die Schweiz ist die Reduktion der CO2-Emissionen in den Industrieländern vorrangig», Pressemitteilung vom 9.11.2000. " $\mathrm{CO}_{2}$-Gesetz tritt in Kraft», Pressemitteilung vom 5.4.2000.

Gesellschaft zur Förderung der schweizerischen Wirtschaft (Wf), Benzin-, Oel- und Gaspreis: «on a déjà donné! ", Information Nr. 11/1, 13.3.2000. Fiscalité verte : le double dividende est un miroir aux alouettes, Information Nr. 12/1, 20.3.2000. La responsabilité individuelle crée un meilleur climat, Dokumentation $\mathrm{Nr}$. 18, 1.5.2000. La loi sur le $\mathrm{CO}_{2}$ plutôt que de nouveaux impôts, communiqué $\mathrm{Nr}$. 18, 1.5.2000.

economiesuisse, $\mathrm{CO}_{2}$ Gesetz: Richtschnur der Schweizer Klimapolitik, Newsletter Nr. 4, Dezember 2000.

\section{INTERNET-ADRESSEN}

Klimakonvention: www.unfccc.int

OACI: www.icao.int

IMO : www.imo.org

IPCC: www.ipcc.ch

Schweizerischer Nationalfonds zur Förderung der wissenschaftlichen Forschung: www.snf.ch

NFP41: www.nfp41.ch

Bundesamt für Energie: www.bfe.admin.ch

Programm EnergieSchweiz: www.EnergieSchweiz.ch (Energie 2000: www.e2000.ch)

28. BWW, EnergieSchweiz geht alle an, Newsletter Nr. 4. 


\subsection{UMSETZUNG DER BIODIVERSITÄTSKONVENTION}

Im Januar 2000 gelang es den Vertragsstaaten der Biodiversitätskonvention nach schwierigen Verhandlungen, ein Protokoll über die biologische Sicherheit $z u$ verabschieden. Die Vertragsparteienkonferenz traf im Mai 2000 zum fünften Mal zusammen. Auf dieser Tagung unterzeichneten rund siebzig Staaten das sogenannte Protokoll von Cartagena. In der Schweiz stellte der Bundesrat dem Parlament den Gen-Lex-Entwurf vor, der die Genmanipulation im ausserhumanen Bereich regeln soll. Die Diskussion in den Eidgenössischen Räten ist für 2001 geplant. Die Frage der Patentierung von Lebewesen, mit der sich die GenLex nicht befasst, wird in der nächsten Revision des Patentgesetzes behandelt; das diesbezügliche Vernehmlassungsverfahren beginnt frühestens im Sommer 2001. Eine Koalition von rund dreissig Entwicklungs-, Landwirtschafts-, Umwelt- und Verbraucherorganisationen hat eine breit angelegte nationale Kampagne unter dem Motto «Keine Patente auf Leben! » gestartet.

\section{$\square$ Das Protokoll von Cartagena}

Die Zielsetzungen der Biodiversitätskonvention umfassen die Bewahrung der biologischen Vielfalt, ihre nachhaltige Nutzung sowie die gerechte und faire Aufteilung der Gewinne aus der Verwendung ihrer genetischen Ressourcen. Ziel ist, ein internationales Abkommen über die biologische Sicherheit, das die wachsenden Bedenken der Bevölkerung wegen potentieller Risiken gentechnisch veränderter Organismen (GVO) aufgreift, in die Konvention aufzunehmen. Eine Sorge gilt der Tatsache, dass zahlreichen Entwicklungsländern die technischen, finanziellen und institutionellen Mittel fehlen, um diese Risiken zu beherrschen; in einigen Ländern ist das nationale Gesetzessystem erst ansatzweise vorhanden. Zwar hatten die zahlreichen Länder, in denen es eine moderne Biotech-Industrie gibt, eine nationale Gesetzgebung eingeführt, aber vor dem Abschluss des Protokolls von Cartagena fehlte doch ein zwingendes internationales Abkommen für Fälle, in denen GVO infolge einer kommerziellen Verwendung oder durch Entweichen bei einem Unfall die nationalen Grenzen überschreiten.

Das Protokoll über die Verhütung biotechnologischer Risiken (oder Protokoll von Cartagena) beruht auf dem Grundsatz der biotechnologischen Sicherheit (biosafety). Der neue Terminus bezeichnet Massnahmen zur Verringerung und Beseitigung potenzieller Risiken der Biotechnologie und ihrer Produkte. Dieses Konzept basiert auf dem Vorsorgeprinzip, wonach das Fehlen wissenschaftlicher Gewissheit in keinem Fall als Vorwand für das Aufschieben von Massnahmen angeführt werden darf, wenn die Gefahr schwerwiegender oder nicht rückgängig zu machender Schäden besteht.

\section{Verfahren zur Erstellung des Protokolls; Art der Verhandlungen}

Artikel 19.3 der Biodiversitätskonvention - eine der Verpflichtungen, welche die Unterzeichnerstaaten eingegangen sind - verlangt von den Parteien, sich mit den Modalitäten eines Protokolls auseinanderzusetzen und dazu ein Verfahren für sichere Voraussetzungen betreffend den Transfer, den Umgang mit und die Verwendung von GVO, die sich möglicherweise schädlich auf die Biodiversität und ihre Bestandteile auswirken können, einzuführen. 
Die Verhandlungen zur Verabschiedung des Protokolls über die biologische Sicherheit erwiesen sich als langwierig und schwierig, konnten jedoch im Januar 2000 in Montreal unter Dach und Fach gebracht werden. Im November 1995 beschloss die Parteienkonferenz, die Verhandlungen zur Ausarbeitung eines Protokolls über die biologische Sicherheit, das anlässlich einer ausserordentlichen Versammlung der Vertragsparteien in Cartagena (Kolumbien) Ende Februar 1999 angenommen werden sollte, zu lancieren. Da die Verhandlungen in eine Sackgasse gerieten, beschloss die Konferenz, die Arbeiten zu unterbrechen und eine neue Verhandlungssitzung vor der im Mai 2000 geplanten fünften Vertragsparteienkonferenz einzuberufen. Die verschiedenen Ländergruppen führten informelle Konsultationen ${ }^{29}$ durch und bekräftigten ihren politischen Willen, ein für alle annehmbares Protokoll zu erarbeiten. In diesen Konsultationen spielte die Schweiz eine Schlüsselrolle. Dank dem Engagement bestimmter Staaten konnten die Parteien zur Abschlusstagung Ende Januar in Montreal eingeladen werden.

$\square$ JSDW 2000, Umsetzung der Biodiversitätskonvention, Aushandlung des Protokolls von Cartagena, Beschreibung der Haltung der Schweiz und der verschiedenen Ländergruppen, die an den Verhandlungen teilnahmen; S. 191-195.

Wiederaufnahme der ausserordentlichen Vertragsparteienkonferenz (Montreal, Januar 2000)

Der zweite Anlauf der ausserordentlichen Vertragsparteienkonferenz zur Annahme des Protokolls über die biologische Sicherheit fand vom 24. bis zum 29. Januar 2000 in Montreal statt. Über 750 Teilnehmer, die 133 Regierungen, NRO, Industriekonzerne sowie Wissenschaftskreise vertraten, beteiligten sich an den Arbeiten. Nach neun harten Verhandlungstagen verabschiedeten die Delegierten das Protokoll von Cartagena über die biologische Sicherheit.

Bestimmte Fragen erwiesen sich in den Verhandlungen als Stolpersteine:

- Geltungsbereich des Protokolls : Einige Teilnehmer wollten den Geltungsbereich auf GVO beschränken, während andere für eine breitere Abdeckung, die auch Agrargüter sowie Nahrungsmittel, Tierfutter oder bei der Herstellung anderer Erzeugnisse verwendete Produkte umfassen sollte.

- Haftung: Wer haftet bei Schäden, die in die Umwelt freigesetzte GVO verursachen?

- Wirtschaftliche und soziale Folgen: Wie lassen sich potenzielle wirtschaftliche und soziale Konsequenzen - Verlust an Wettbewerbsfähigkeit der traditionellen Kulturen im Vergleich zu GVO-Importen - eindämmen?

口 Vereinbarkeit mit anderen internationalen Abkommen: Das Problem der Beziehungen zwischen dem Protokoll und anderen internationalen Abkommen, insbesondere jenen der WTO, muss geregelt werden.

\section{Ziele und Inhalt des Protokolls}

Das Protokoll dient als internationaler Gesetzesrahmen im Bereich der biotechnologischen Sicherheit. Es soll gewährleisten, dass durch die moderne Biotech-

29. Der kolumbische Umweltminister Juan Mayr Maldonado, Präsident der ausserordentlichen Versammlung, organisierte im Juli in Montreal und im September 1999 in Wien zwei halb offizielle Konsultationsrunden, die allen interessierten Delegationen offen standen 
nologie veränderte lebende Organismen, welche eine potenzielle Gefahr für die Erhaltung und nachhaltige Nutzung der Artenvielfalt in sich bergen, unter sichersten Voraussetzungen transportiert, gehandhabt und verwendet werden.

Das Protokoll von Cartagena befasst sich mit den Voraussetzungen für den Transfer, die Handhabung und die Verwendung von GVO, welche die Artenvielfalt möglicherweise beeinträchtigen; besondere Aufmerksamkeit gilt den grenzüberschreitenden Bewegungen, die nur selten in nationalen Regelungen erfasst sind. Das Protokoll sieht das Verfahren der vorherigen Zustimmung in Kenntnis der Sachlage (AIA ${ }^{30}$ ) vor, das für die erste grenzüberschreitende Bewegung von GVO zum direkten Einsatz in der Umwelt, aber auch für die Einfuhr von landwirtschaftlichem Rohmaterial mit GVO (zwecks Verbrauch oder Verarbeitung) gilt. Im Rahmen dieses Verfahrens erhalten die Einfuhrländer eine Notifizierung mit allen notwendigen Informationen, um Umwelt- und Gesundheitsrisiken noch vor dem Import beurteilen zu können. Das Protokoll anerkennt das Vorsorgeprinzip im Entscheidungsprozess. Ein weiterer Fortschritt wird bei der Kennzeichnung zur Identifizierung von GVO erzielt: Die Bestimmungen zu GVO, die zur Freisetzung in der Umwelt bestimmt sind, müssen sehr detailliert abgefasst werden. Dagegen sprachen sich die GVO-Produzentenländer (im Mittelpunkt die Vereinigten Staaten und Kanada) sowie der Block Europäische Union / Länder des Südens gegen die Beschriftung von zum Verbrauch oder zur Verarbeitung bestimmten Ladungen, die möglicherweise gentechnisch veränderte Organismen enthalten, aus. Schliesslich wurde mit der Formulierung «kann GVO enthalten » ein nur teilweise befriedigender Kompromiss gefunden.

Das Protokoll sieht vor, einen «Biosafety Clearing House Mechanism» zu schaffen, um den internationalen Informationsaustausch zur Umwelt- und Gesundheitssicherheit der Biotechnologien zu fördern. Ausserdem soll gemäss dem Protokoll die Zusammenarbeit zwischen Norden und Süden insbesondere auf den Gebieten der Wissenschaft, Technik und der Institutionen vertieft werden. Das Protokoll besitzt den gleichen Status wie die übrigen handelsbezogenen internationalen Abkommen, die sich gegenseitig in ihren Zielsetzungen unterstützen sollen. Es handelt sich um einen wichtigen Punkt, der sicherlich zur Klärung des Verhältnisses zwischen der WTO und den multilateralen Umweltabkommen beitragen wird ${ }^{31}$.

\section{Zwischenstaatlicher Ausschuss für das Cartagena-Protokoll}

Die Parteienkonferenz setzte ausserdem den zwischenstaatlichen Ausschuss für das Cartagena-Protokoll (ICCP) ein, der folgende Aufgaben erfüllt:

- Schaffung eines Informationsaustausch-Zentrums über biologische Sicherheit (Biosafety Clearing House Mechanism);

口 Erstellung eines Programms für die Entwicklungsländer, um sie mit den erforderlichen technischen und institutionellen Kapazitäten zur Protokollratifizierung auszustatten;

- Vorbereitung der Beschlüsse für die erste Tagung der Vertragsparteien des Protokolls.

30. In den meisten Texten zum Verfahren der vorherigen Zustimmung in Kenntnis der Sachlage wird die englische Abkürzung AIA verwendet (Advanced Informed Agreement).

31. BUWAL, Gentechnologie heute, Faktenblatt Nr. 16, Cartagena-Protokoll. Verfügbar am Internet-Site des BUWAL. 
Die erste Versammlung fand im Dezember 2000 in Montpellier (Frankreich) statt. Die Delegierten, die an der ersten Tagung des zwischenstaatlichen Ausschusses teilnahmen, einigten sich auf die Errichtung eines Informationsaustausch-Zentrums zur Verhütung biotechnologischer Risiken, fassten jedoch keine Beschlüsse, weder zur Frage der Kennzeichnung von GVO noch zu etwaigen Sanktionen gegen Staaten, die sich nicht an das Protokoll halten. Die nächste Tagung des ICCP soll im Oktober 2001 in Montreal stattfinden.

\section{Position der Schweiz}

Die Schweiz hatte seit Verhandlungsbeginn «eine kritische und zugleich konstruktive Haltung ${ }^{32}$ eingenommen. Sie ergriff die Initiative zur Schaffung einer Kompromissgruppe, bestehend aus Japan, Norwegen, Mexiko und Südkorea, die es möglich machte, dass das Protokoll nach fünfjährigen Verhandlungen angenommen werden konnte. Die wesentliche Rolle der Schweiz beim Abschluss der Verhandlungen wurde mehrfach anerkannt ${ }^{33}$. Nach Abschluss der Konferenz von Montreal äusserte sich die Schweizer Delegation sehr erfreut über die Verabschiedung des Protokolls und über den Kompromiss zwischen den Erfordernissen des Umweltschutzes und den Interessen des Welthandels ${ }^{34}$.

\section{$\square$ Fünfte Vertragsparteienkonferenz (Mai 2000)}

Die fünfte Vertragsparteienkonferenz zur Biodiversitätskonvention (CoP-5) fand vom 15. bis zum 26. Mai 2000 am Sitz des Umweltprogramms der Vereinten Nationen in Nairobi statt. 179 Länder ratifizierten die Konvention ${ }^{35}$. Die Vereinigten Staaten gehören nicht dazu; sie besitzen lediglich einen Beobachterstatus. Als Höhepunkt der Versammlung wurde das Cartagena-Protokoll anlässlich einer hochrangigen Sitzung zur Unterzeichnung freigegeben. Die Versammlung behandelte eine mit rund dreissig Punkten befrachtete Tagesordnung.

Die Schweizer Delegation unter der Leitung von Botschafter Beat Nobs bestand aus Vertretern des BUWAL, des seco, des Bundesamts für Landwirtschaft, des Eidgenössischen Instituts für geistiges Eigentum (IGE) sowie aus zwei NichtRegierungsvertretern (eine NRO, Swissaid, und ein Vertreter der Privatwirtschaft, Novartis).

\section{Unterzeichnung des Cartagena-Protokolls}

Das Protokoll von Cartagena wurde auf einer hochrangigen Sitzung am 24. Mai 2000 den Vertragsparteien zur Unterzeichnung freigegeben. 80 Konventionsparteien, darunter die Schweiz und alle Staaten der Europäischen Union, unterzeichneten das Protokoll ${ }^{36}$, das 90 Tage nach der 50. Ratifizierung in Kraft treten wird. Im Dezember 2000 hatten erst zwei Staaten - Bulgarien sowie Trinidad und Tobago - das Protokoll ratifiziert. Der Bundesrat plädiert für eine möglichst

32. BUWAL, «Weltweite Regelung für den Export gentechnisch veränderter Organismen», Pressemitteilung, 12. Januar 2000.

33. La Liberté, Artikel über die Rolle der Schweiz bei der Aushandlung des Cartagena-Protokolls, 3. Februar 2000.

34. BUWAL, «Montreal: Ein Erfolg, Weltweite Regelung für den Export von gentechnisch veränderten Organismen », Pressemitteilung, 29. Januar 2000.

35. Stand vom November 2000.

36. Stand vom Dezember 2000. 
rasche Ratifizierung, wenn machbar vor Ende 2001, damit die Schweiz beim Inkrafttreten des Protokolls Vertragspartei ist. Die Ratifizierung setzt keine Gesetzesänderungen voraus.

\section{Übrige Tagesordnungspunkte der CoP-5}

Die Delegierten der CoP-5 untersuchten und genehmigten rund dreissig Beschlüsse. Die wichtigsten darunter sind ${ }^{37}$ :

- Zugang zu genetischen Ressourcen und Weitergabe der Vorteile, die sich aus ihrer Nutzung ergeben;

๖ nachhaltige Nutzung der Artenvielfalt mit besonderem Schwerpunkt auf dem Fremdenverkehr;

」 Definition einer Globalstrategie für die Erhaltung der Pflanzen;

- Globale Initiative für Taxonomie (GTI);

๑ Überarbeitung des Arbeitsprogramms betreffend marine Ökosysteme und Binnengewässer sowie die Artenvielfalt in der Land- und Forstwirtschaft ;

๑ traditionelle Kenntnisse;

ป wissenschaftliche und technische Zusammenarbeit;

$\checkmark$ bestimmte administrative und finanzielle Gesichtspunkte: Funktionsweise der Konvention, Tätigkeiten des Globalen Umweltfonds (GEF - Finanzmechanismus der Konvention), Haushalt des Sekretariats.

\section{Position der Schweiz}

Die Schweiz setzte sich unter den verschiedenen Punkten nachdrücklich für konkrete Massnahmen ein, um vor allem den Ländern des Südens den Zugang zu genetischen Ressourcen und das Teilen der Vorteile aus ihrer Nutzung zu erleichtern. Die Entwicklungsländer fordern nämlich als Gegenleistung zum Schutz und zur nachhaltigen Verwendung der Artenvielfalt, dass sie ebenfalls am daraus entstehenden Nutzen teilhaben können. Die Schweiz legte einen Leitlinienentwurf vor, den das seco, das Eidgenössische Institut für geistiges Eigentum und das BUWAL vorbereitet hatten. Der Vorschlag zielt darauf $a b$, die Entwicklungsländer zu unterstützen und ihnen bei der Einführung von Bewilligungsverfahren für die Nutzung der genetischen Ressourcen zu helfen. Klar abgegrenzte Etappen und Verantwortlichkeiten ermöglichen eine bessere Zusammenarbeit unter Regierungen, Industrie, Forschung, internationalen Organisationen und Zivilgesellschaft. Der Leitlinienentwurf deckt die verschiedenen Demarchen bis zur Vermarktung ab. Der Vorschlag stiess auf grosse Resonanz. Eine Arbeitsgruppe wurde beauftragt, die Leitlinien $\mathrm{zu}$ formulieren ${ }^{38}$. Ausserdem forderte die Schweiz eine Intensivierung der Arbeiten der Konvention, um die Anliegen der Artenvielfalt in den sektoriellen Politiken - vor allem im Agrar- und Forstbereich - stärker zu verankern ${ }^{39}$.

37. Zahlreiche Referenzdokumente zur CoP-5 befinden sich an folgender Adresse: www.biodiv.org/cop5/index-html

38. «Der weite Weg von der Biopiraterie zur fairen Nutzung», in Umwelt +, Dossier «Neuland Gentechnologie», BUWAL, 4/2000.

39. BUWAL, Pressemitteilung, «Regelungen für die Nutzung der genetischen Ressourcen» (3. Mai 2000) und «Die Schweiz unterzeichnet das Protokoll über die biologische Sicherheit in der Biotechnologie» (26. Mai 2000) 
Die Schweiz hatte an den Diskussionen mehrerer Arbeitsgruppen aktiv mitgewirkt (Teilen der Vorteile, Wälder, Verhältnis der Konvention zu den anderen Institutionen, Haushalt, Finanzmechanismus und Arbeitsprogramm) und äusserte sich zufrieden über die Ergebnisse der Konferenz.

Die sechste Vertragsparteienkonferenz wird im zweiten Quartal 2002 in Den Haag (Niederlande) stattfinden.

\section{$\square$ Gen-Lex-Vorlage}

\section{Hauptelemente von Gen-Lex}

Im Januar 2000 legte der Bundesrat die allgemeinen Grundsätze fest, auf denen die Gen-Lex-Vorlage beruht, welche die gesetzlichen Leitplanken im Bereich der ausserhumanen Gentechnik verstärken soll ${ }^{40}$. Die Gen-Lex konkretisiert den Artikel 120 der neuen Bundesverfassung (bisher Artikel 24novies) und beinhaltet vor allem eine Änderung des Umweltschutzgesetzes, aber auch verschiedener anderer Gesetze (Tierschutzgesetz und Landwirtschaftsgesetz). Die wesentlichen Ziele der neuen Bestimmungen für die Gentechnologie sind: Schutz von Mensch und Umwelt, Erhaltung und nachhaltige Nutzung der Artenvielfalt, Achtung der Würde der Kreatur. Am 1. März 2000 genehmigte der Bundesrat die an das Parlament gerichtete Gen-Lex-Botschaft ${ }^{41}$. In seiner Vorlage sah der Bundesrat davon ab, die GVO zu verurteilen, und entschied sich statt dessen dafür, ihre Verwendung einer Bewilligungspflicht zu unterstellen. Die Idee eines Moratoriums wurde abgelehnt. Gleichzeitig trifft aber die Regierung verschiedene Sicherheitsvorkehrungen wie die Verschärfung der Haftpflicht für Saatguthersteller, die Verlängerung der Verjährungsfrist (von 20 auf 30 Jahre) und das Verbot von «Terminator»-Saatgut. Der Bundesrat wünscht grössere Transparenz für die Verbraucher; die Vorlage enthält denn auch Hinweise zur Deklarationspflicht für Produkte, die GVO enthalten können. Wie in der Presse berichtet wurde, war die Regierung zur Gen-Lex-Vorlage gespalten: Moritz Leuenberger wünschte ein restriktiveres Projekt, während Pascal Couchepin schliesslich eine liberalere Lösung durchsetzte ${ }^{42}$.

\section{Reaktionen auf die Gen-Lex-Vorlage}

Die Vorlage löste zahlreiche Reaktionen aus, in denen sich verschiedene Fronten abzeichneten. Die bürgerlichen Parteien (SVP, FDP und CVP) begrüssten die Entscheidung des Bundesrates, während die Grünen und die Sozialdemokraten die Freisetzung von GVO nach wie vor bekämpfen wollen. Pascal Couchepin spricht von einem Sieg für die Wirtschaft, aber in den Wirtschaftskreisen herrschen gemischte Gefühle. Nach Auffassung von Novartis bedeutet die Ausweitung der Haftpflicht einen Schritt in die richtige Richtung, während der Pharmazeutenverband Interpharma diesen Punkt zur Diskussion stellen möchte. Die Versicherer kritisieren die Verlängerung der Verjährungsfrist und bedauern, dass die Saatguthersteller sich dem Risiko von Gerichtsprozessen aussetzen müssen. Der Vorort teilt diese Sichtweise und ist der Auffassung, dass das Opfer den direkten Kausalzusammenhang zwischen dem Schaden und einem mit Mängeln

40. UVEK, Pressemitteilung, Neue Leitplanken für die Gentechnologie, 19. Januar 2000.

41. Botschaft zu einer Änderung des Bundesgesetzes über den Umweltschutz (USG) vom 1. März 2000, Bundesblatt vom 25. April 2000. Botschaft 00.008.

42. Le Temps, «Des garde-fous pour les OGM mais pas de moratoire», 20. Januar 2000. 
behafteten Produkt nachweisen soll43. Die Umweltkreise wie Pro Natura, kleinere und mittelgrosse Landwirte, Bio Schweiz und Greenpeace protestierten und wandten ein, die Regierung habe die schweizerische Landwirtschaft geopfert und ihr die Chance verbaut, sich international durch eine ökologische Produktion ohne GVO zu profilieren ${ }^{44}$. Im April 2000 sprach sich der Schweizerische Bauernverband (SBV) für ein Moratorium von 10 Jahren aus ${ }^{45}$.

Im August 2000 setzte der Bundesrat im Zusammenhang mit der Gen-Lex-Botschaft eine neue interdepartementale Arbeitsgruppe ein, in welcher dreizehn Bundesdienste vertreten sind (IDAGEN). Die IDAGEN hat folgende Aufgaben: Ausarbeitung einer Gesamtstrategie des Bundesrates für den Bereich Gentechnologie, Diskussion der Risikoforschung in der Gentechnologie, Austausch von Informationen über den künftigen Rechtsetzungsprozess und über den Vollzug der Gen-Lex-Vorlage usw. ${ }^{46}$.

Im Laufe des Jahres 2000 wurde die Gen-Lex-Vorlage von der Kommission für Wissenschaft, Bildung und Kultur (WBK) des Ständerates geprüft. Nicht weniger als sechs Sitzungen brauchte die Kommission, um sich mit der «umfassenden und facettenreichen ${ }^{47}$ Vorlage abschliessend auseinanderzusetzen. Die Kommission verlangte eine zweite Lesung, da viele Beschlüsse erst provisorisch getroffen oder im Hinblick auf eine vertiefte Prüfung zurückgestellt worden waren. Ausstehend ist zum Beispiel die heikle Frage eines Moratoriums für die Freisetzung von transgenen Pflanzen und Tieren. Die Eidgenössischen Räte sollen sich im Laufe des Jahres 2001 mit der Gen-Lex-Vorlage befassen.

\section{$\square$ Patentierung von Lebewesen}

\section{Revision des Bundesgesetzes über Patente}

Auf die Frage der Patente wird in der Gen-Lex-Vorlage, die in die Vernehmlassung gegeben wurde, nicht eingegangen. In der Schweiz sind Pflanzen und Tiere zwar grundsätzlich nicht patentierbar, aber es ist möglich, auf mehrere Arten anwendbare genetische Erfindungen durch ein Patent zu schützen. Die jüngste, noch laufende Revision des Patentgesetzes wurde im Anschluss an die Motion Leumann (98.3243) in die Wege geleitet. Die Motion fordert die Regierung auf, das Patentgesetz an die europäische Richtlinie über den rechtlichen Schutz biotechnologischer Erfindungen anzupassen ${ }^{48}$. Seit Juli 1998 erlaubt die Richtlinie Patente auf Gene und Gensequenzen, Zellen, Gewebe, Pflanzen, Tiere, menschliche Organe und bestimmte menschliche Embryonen. Erst nach zehn Jahren zäher Verhandlungen konnte die Richtlinie, welche die Mitgliedsstaaten bis spätestens am 30. Juli 2000 in die nationale Gesetzgebung umsetzen müssen, verabschiedet werden. Nur zwei Staaten (Dänemark und Finnland) haben ihre Gesetzgebung angepasst, während die Vertreter Frankreichs und Deutschlands die europäische Richtlinie kritisierten. Die Niederlande und Italien legten sogar Beschwerde beim Europäischen Gerichtshof ein ${ }^{49}$.

43. Le Temps, «Des garde-fous pour les OGM mais pas de moratoire», 20. Januar 2000.

44. Dépêche AP, «Pas d'interdiction ni de moratoire sur les OGM», 12. Januar 2000.

45. L'Agefi, «L'USP pour un moratoire jusqu'en $2010 », 27$. April 2000.

46. UVEK, Medienmitteilung, Gentechnologie: Der Bundesrat setzt eine neue interdepartementale Arbeitsgruppe (IDAGEN) ein, 30. August 2000.

47. Parlamentsdienste, Pressemitteilung, «Gen-Lex: Es wird Frühling werden», 8. November 2000.

48. Europäische Richtlinie 98/44/EC.

49. Greenpeace «Gravierende Mängel der Gen-Patent-Richtlinie 98/44/EC» in Presseunterlagen «Keine Patente auf Leben! ", 4. September 2000. 
Das Vernehmlassungsverfahren zur Revision des Bundesgesetzes über Patente war für Sommer 2000 geplant gewesen, musste jedoch aus verschiedenen Gründen - unter anderem wegen der jüngsten Annahme des Patentrechtvertrags (WIPO) und wegen der Überarbeitung des Europäischen Patentübereinkommens - vertagt werden ${ }^{50}$. Das Eidgenössische Institut für geistiges Eigentum (IGE), das den Gesetzesentwurf und den erläuternden Bericht für die Vernehmlassung vorbereiten soll, möchte die Entwicklungen im Vollzug der Richtlinie der Europäischen Union über den rechtlichen Schutz biotechnologischer Erfindungen abwarten, bevor entsprechende Änderungen für die Schweiz beschlossen werden. Sobald das IGE über die erforderlichen Informationen verfügt, sollen die Gesetzesarbeiten beginnen. Die externe Vernehmlassung bei den interessierten Kreisen wird nach der Ämterkonsultation des Revisionsentwurfs und nach der Genehmigung durch den Bundesrat, frühestens jedoch im Sommer 200151, beginnen.

\section{Nationale Kampagne "Keine Patente auf Leben!»}

Rund dreissig Organisationen aus den Bereichen Zusammenarbeit und Entwicklung, Umwelt, Verbraucher, Bauernkreise, darunter Swissaid, die Erklärung von Bern, Greenpeace und der WWF52 starteten im September 2000 eine Kampagne unter dem Motto «Keine Patente auf Leben!». Die Koalition dieser Verbände wehrt sich gegen die Revision des Bundesgesetzes über Patente, worin Patente auf Lebewesen bewilligt werden sollen. Die NRO argumentieren, der Bundesrat wolle Gesetzesgrundlagen für die Angleichung des schweizerischen Gesetzes an die Europäische Richtlinie 98/44/EG schaffen, obwohl doch die EU-Mitgliedstaaten selbst grösste Bedenken hegten (vgl. oben).

Die Argumente gegen die Patentierung von Lebewesen beruhen auf ethischen Beweggründen, auf der Furcht vor einem Monopol der Agrarindustriekonzerne auf Saatgut und vor einer Plünderung der Biodiversität im Süden. Die Sorgen der NRO gelten auch der Forschung ; sie befürchten, dass Patente auf Leben die Produktentwicklung in der Landwirtschaft und im pharmazeutischen Bereich jahrelang blockieren könnten. Schliesslich mahnen die NRO die Verpflichtungen der Staaten im Rahmen der Biodiversitätskonvention an: Danach müssen kommerzielle oder sonstige Vorteile aus genetischen Ressourcen gleichmässig verteilt und der Zugang zu Biotechnologien für die Länder des Südens erleichtert werden. Nach ihrer Auffassung steht die Patentgewährung im Widerspruch zu allen diesen Anforderungen.

Die Kampagne appelliert an die Bevölkerung, der die Problematik am Herzen liegt, sich damit an den Bundesrat zu wenden, genauer gesagt eine Postkarte an Ruth Metzler zu adressieren und sie zu ersuchen, alles daran zu setzen, um Patente auf Leben zu verhindern.

50. Im November 2000 tagte in München die diplomatische Konferenz zur Überarbeitung des Europäischen Patentübereinkommens. Die diplomatische Konferenz nahm knapp 30 Jahre nach Abschluss des Übereinkommens dessen vollständige Revision in Angriff. Als Vorsitzender amtierte Roland Grossenbacher, der Direktor des Eidgenössischen Instituts für geistiges Eigentum. Allerdings wurde die Frage der Patentierung von biotechnologischen Erfindungen absichtlich aus der aktuellen Revision ausgeklammert, zumal es dazu in der Europäischen Union noch keinen Konsens gibt.

51. Eidgenössisches Institut für geistiges Eigentum, Jahresbericht 1999/2000, Bern, November 2000.

52. Die Kampagne «Keine Patente auf Leben» wird präsentiert am Site von Swissaid: www.swissaid.ch/ politik.htm 


\section{QUELLEN}

Protokoll von Cartagena über die Verhütung biotechnologischer Risiken.

Botschaft zu einer Änderung des Bundesgesetzes über den Umweltschutz (USG) vom 1. März 2000. Botschaft 00.008 .

Zusammenfassung der wiederaufgenommenen Sondertagung der Vertragsparteienkonferenz für die Annahme des Biosicherheitsprotokolls - 24.-28. Januar 2000, in Earth Negotiation Bulletin, Band 9, Nr. 137.

Fünfte Vertragsparteienkonferenz zur Biodiversitätskonvention, 15.-26. Mai 2000, in Earth Negotiation Bulletin, Band 9, Nr. 160.

A. Crosbey and S. Burgiel, «The Cartagena Protocol on Biosafety: an analysis of results», in IISD Briefing Note, 2000. (www.iisd.ca).

Eidgenössisches Institut für geistiges Eigentum, Jahresbericht 1999/2000, Bern, November 2000.

Bundesamt für Umwelt, Wald und Landschaft (BUWAL), Umwelt, Dossier «Biotechnologie: Chancen und Risiken », 4/2000.

Bundesamt für Umwelt, Wald und Landschaft (BUWAL), Umwelt, Dossier «Neuland Gentechnologie», $4 / 2000$.

Umweltprogramm der Vereinten Nationen, Press release 2000.

Sekretariat der Konvention über die biologische Vielfalt, Press release, Nairobi 2000 - Fifth Meeting of the Conference of the Parties Convention on Biological Diversity.

Bundesamt für Umwelt, Wald und Landschaft (BUWAL), Pressemitteilungen 2000.

\section{INTERNET-ADRESSEN}

Offizieller Site der Biodiversitätskonvention: www.biodiv.org

Cartagena-Protokoll über biologische Sicherheit: www.biodiv.org/biosafe/Protocol/Index.html

Bundesamt für Umwelt, Wald und Landschaft (BUWAL): www.buwal.ch/index.htm

BUWAL zur Thematik «Biotechnologie»: www.buwal.ch/stobobio/biotechnologie/index.htm

Eidgenössisches Institut für geistiges Eigentum: www.ipi.ch

International Institute for Sustainable Development (IISD), Zusammenfassung der Verhandlungen über die biologische Sicherheit: www.iisd.ca/biodiv/excop

\subsection{UMWELTDIPLOMATIE DER SCHWEIZ}

Seit dem Erdgipfel von Juni 1992 haben sich Umweltfragen zu einem zentralen Aspekt der multilateralen Zusammenarbeit entwickelt. Die Staaten stehen in der Pflicht, die ratifizierten Umweltübereinkommen durchzuführen, und beteiligen sich an den Vertragsparteienkonferenzen über die geltenden Konventionen, wie der Konvention über den Klimawandel, über die Biodiversität, die Wüstenbildung und die Sonderabfälle. In den nächsten Jahren werden neue multilaterale Abkommen in Kraft treten (Konvention über persistente organische Schadstoffe $(P O P)$ und Konvention über den internationalen Handel mit Chemikalien (PIC)) und so das juristische Arsenal im Umweltbereich vervollständigen. Ausserdem finden jedes Jahr regelmässige Versammlungen statt, auf denen die Regierungen angespornt werden, die notwendigen Massnahmen für die nachhaltige Entwicklung zu ergreifen (z.B. Kommission für nachhaltige Entwicklung, Globaler Umweltfonds GEF). Einige Tagungen sind besonderen Themen gewidmet; im Jahr 2000 waren es zwei wichtige Treffen, das Zwischenstaatliche Forum über Wälder (Intergovernmental Forum on Forests), das einige wegweisende Beschlüsse gefasst hat, und das Globale Wasserforum. Die Schweiz betreibt in der Umweltdiplomatie eine rege Tätigkeit. Sie nimmt regelmässig an den Vertragsparteienkonferenzen teil, wirkt aktiv an den Verhandlungen neuer Konventionen mit und bewirbt sich um den Sitz deren Sekretariate. Die Schweiz hat einen ständigen Sitz im GEF-Exekutivrat inne und vertritt dort seit 2000 Aserbaidschan, Kirgisistan, Tadschikistan, Turkmenistan und Usbekistan. An allen 
Umweltkonferenzen, über die in der diesjährigen Ausgabe des Jahrbuchs Schweiz-Dritte Welt berichtet werden soll, hat die Schweiz engagiert teilgenommen. Die Umsetzung der Konvention über den Klimawandel und der Konvention über die Biodiversität wird im vorliegenden Band in getrennten Artikeln behandelt.

\section{$\square$ Vertragsparteienkonferenzen der Umweltübereinkommen}

\section{Basler Übereinkommen über gefährliche Abfälle - Dezember 1999}

Die Stadt Basel beherbergte vom 6. bis zum 10. Dezember 1999 die fünfte Konferenz der Vertragsparteien des Basler Übereinkommens über gefährliche Abfälle. Im Vordergrund der Tagung standen die Annahme des Protokolls von Basel über die internationale Haftung beim Transport von gefährlichen Abfällen sowie die Feierlichkeiten zum 10. Jahrestag des Konventionsabschlusses in der Rheinstadt. Zum Vorsitzenden der Konferenz wurde BUWAL-Direktor Philippe Roch gewählt. In seiner Rede erwähnte er die Absicht, ein Arbeitsprogramm zur Unterstützung der Entwicklungsländer zu erstellen: Durch die Förderung des Technologietransfers soll die Produktion von Sonderabfällen in den Entwicklungsländern begrenzt und die umweltgerechte Entsorgung ermöglicht werden. Das Sekretariat der Konvention ist seit 1993 in Genf in der Schweiz untergebracht.

Das Basler Protokoll sieht eine umfassende Haftungsregelung für internationale Transporte gefährlicher Abfälle vor. Der Exporteur haftet für den Transport, bis die Abfälle dem Entsorgungsunternehmen im Importland vorschriftsmässig übergeben werden. Ab diesem Zeitpunkt haftet das Entsorgungsunternehmen. Für Fälle, in denen der Schadensverursacher der Verantwortung aus seiner Haftpflicht nicht nachkommen kann, wird ein Fonds eingerichtet. Die neue Regelung ist vor allem für Entwicklungsländer wichtig, da sie im Unterschied zu den Industrieländern nicht immer über hinlängliche Haftpflichtgesetze verfügen. Die Regelung wurde auf der Konferenz von Basel über gefährliche Abfälle im Dezember 1999 von 115 Ländern angenommen. Erstmals wurden Haftpflichtvorschriften auf internationaler Ebene in einem Umweltabkommen von grosser Bedeutung niedergelegt.

Das Basler Protokoll ergänzt das 1989 abgeschlossene Basler Übereinkommen über die Kontrolle der grenzüberschreitenden Verbringung gefährlicher Abfälle und ihrer Entsorgung. Die Schweiz hatte damals die Ausarbeitung des Übereinkommens massgeblich mit bestimmt.

Am 10. März 2000 unterzeichnete Bundesrat Moritz Leuenberger das Protokoll. Damit hat die Schweiz als erstes Land den Text paraphiert, der nun noch vom Parlament ratifiziert werden muss.

凹SDW 1999, Hintergrund und Ziele des Basler Übereinkommens, Vierte Vertragsparteienkonferenz, S. 149-151.

UNEP, Basel Convention Press Kit, Managing Hazardous Waste, Dezember 1999.

BUWAL, Sonderabfälle in der Schweiz. Vom Problem zur Lösung, Broschüre zum zehnten Jahrestag des Basler Übereinkommens, Bern, 1999. Internet-Site des Basler Übereinkommens: www.basel.int

\section{Übereinkommen zur Bekämpfung der Wüstenbildung - Dezember 2000}

Die vierte Vertragsparteienkonferenz der Konvention gegen die Wüstenbildung wurde vom 11. bis zum 22. Dezember 2000 in Bonn abgehalten. Die schweize- 
rische Delegation wurde von H.-P. Cart, Vizedirektor der DEZA, geleitet und setzte sich aus Vertretern der DEZA, der Politischen Abteilung V des EDA und der Arbeitsgemeinschaft der Hilfswerke zusammen.

Die Konferenz widmete sich hauptsächlich der Lageuntersuchung in den Ländern Asiens und Lateinamerikas und prüfte, wie es den von der Wüstenbildung betroffenen Ländern bisher gelungen ist, die Konvention umzusetzen.

Die DEZA fördert Projekte, die zur Bekämpfung der Wüstenbildung beitragen. Häufig handelt es sich dabei um Aktionen zur Förderung der nachhaltigen Boden- und Wasserbewirtschaftung, wobei geeignete Produktions- und Bodennutzungssysteme zum Einsatz kommen (vor allem im Sahel, in den Trockengebieten Asiens und in der Andenregion in Lateinamerika). In Asien investierte die DEZA zusammen mit den schweizerischen Hilfswerken zwischen 1997 und 200062 Millionen Franken in Projekte zur Bekämpfung der Wüstenbildung. Für Lateinamerika wurden im gleichen Zeitraum 32 Millionen Franken aufgewendet.

@ JSDW 2000, Umsetzung des Übereinkommens zur Bekämpfung der Wüstenbildung, S. 196-200.

Offizieller Site des Übereinkommens zur Bekämpfung der Wüstenbildung: www.unccd.de

\section{Protokoll von Montreal zum Schutz der Ozonschicht - Dezember 2000}

Die zwölfte Vertragsparteienkonferenz des Montrealer Protokolls (CoP-12) tagte vom 11. bis zum 15. Dezember 2000 in Ouagadougou (Burkina Faso). Das Ozonloch über der Antarktis erreichte in diesem Jahr die Rekordausdehnung von 28 Millionen km2 - zweimal die Fläche Europas. Zum ersten Mal sind bewohnte Gebiete in Chile (Feuerland) betroffen. Anlässlich der CoP-12 plädierte die von Botschafter Beat Nobs (BUWAL) geleitete schweizerische Delegation für die Annahme neuer Massnahmen, um die Wiederherstellung der Ozonschicht zu beschleunigen.

Offizieller Site des Protokolls von Montreal: http://www.unep.ch/ozone/

\section{$\square$ Zwei neue Übereinkommen über chemische Produkte}

Konvention über persistente organische Schadstoffe

Das Problem des Umgangs mit chemischen Produkten gewinnt international an Bedeutung und ist für die Entwicklungsländer von grösstem Interesse. Die globalen, unter der Ägide des Umweltprogramms der Vereinten Nationen (UNEP) begonnenen Verhandlungen wurden im Dezember 2000 in Johannesburg abgeschlossen, nachdem die Delegationen aus über 120 Ländern ein neues Übereinkommen über organische Schadstoffe und über persistente organische Schadstoffe (POPs) verabschiedeten. Die schweizerische Delegation unter der Leitung des BUWAL wirkte ebenfalls an den Vorbereitungsarbeiten mit und unterstützt Projekte im Bereich der persistenten toxischen Stoffe.

In den Verhandlungen ging es um die Umsetzungsmodalitäten, die Finanzierung für die Entwicklungsländer und um das Verfahren zum Verbot neuer gefährlicher Produkte. Das rechtlich bindende völkerrechtliche Instrument soll die Herstellung und Verwendung bestimmter besonders gefährlicher toxischer Produkte verbieten (in der Schweiz sind die Produktion, Verwendung und der Handel mit POPs bereits seit mehreren Jahren verboten). Das Übereinkommen wird im Laufe des Jahres 2001 den Staaten zur Unterzeichnung vorgelegt. 


\section{Übereinkommen über den internationalen Handel mit Chemikalien}

Dank einer neuen internationalen Konvention sollen künftig die Risiken gefährlicher chemischer Stoffe für Umwelt und Gesundheit reduziert werden. Bislang bestand vor allem in den Entwicklungsländern keine Gewähr für eine sichere Verwendung solcher Produkte. Nach dem neuen Übereinkommen dürfen die Exporte künftig nur bewilligt werden, wenn das Empfängerland über das Gefahrenpotenzial der Produkte vorher informiert wurde und der Einfuhr zugestimmt hat.

Die Schweiz hat diese Konvention vor zwei Jahren in Rotterdam unterzeichnet. Der Bundesrat hat nun dem Parlament mit Blick auf die Ratifizierung eine Botschaft vorgelegt. Die Konvention tritt in Kraft, sobald sie von 50 Ländern ratifiziert worden ist.

Für die fraglichen Substanzen ${ }^{53}$ gilt der Grundsatz der vorherigen Zustimmung nach Inkenntnissetzung (Prior Informed Consent PIC). Nach diesem Prinzip wurde denn auch die Konvention benannt (PIC-Übereinkommen von Rotterdam). Für alle Stoffe auf der PIC-Liste wird ein Bericht veröffentlicht, der Aufschluss über die Gefährlichkeit und über die erforderlichen Sicherheitsmassnahmen gibt. Die Vertragsparteien entscheiden gestützt auf den Bericht, ob sie etwaige Einfuhren einer PIC-Substanz bewilligen oder verbieten. Ihre Entscheidungen werden zusammengestellt und veröffentlicht. Die Substanzen dürfen nicht exportiert werden, wenn das Empfängerland nicht einverstanden ist.

Die Konvention, die das UNEP und die FAO vorbereitet hatten, beruht auf einem bereits existierenden, freiwilligen Verfahren. Für die Schweiz mit ihrer exportorientierten und volkswirtschaftlich wichtigen Chemieindustrie spielt das Übereinkommen eine wesentliche Rolle. Die chemische Industrie hatte noch vor Abschluss der Arbeiten ihre Verantwortung gegenüber den Entwicklungsländern wahrgenommen, indem sie einen Verhaltenskodex ausarbeitete und sich darin verpflichtete, die Regeln des freiwilligen Verfahrens zu befolgen.

Die Schweiz hat ihre Kandidatur als Sitzstaat für die Sekretariate der beiden neuen Konventionen eingereicht, um die wichtigsten internationalen Instanzen auf dem Gebiet der chemischen Stoffe in Genf zu vereinen. Die Entscheidungen werden anlässlich der nächsten Vertragsparteienkonferenzen - voraussichtlich 2003 für die PIC-Konvention, 2005 für die POP-Konvention - gefällt.

Site des Umweltprogramms der Vereinten Nationen für Fragen zur Chemie: http://irptc.unep.ch/

BUWAL, Pressemitteilung, "Verbot oder starke Reduktion von DDT, PCB und anderen persistenten organischen Schadstoffen weltweit in Sicht», 30. November 2000.

53. Zu den Substanzen gehören:

- das Insektizid DDT und die PCB (polychlorierte Biphenyle), die in Transformatoren und Kondensatoren verwendet werden, sich in der Umwelt anreichern und die Reproduktionsfähigkeit bestimmter Organismen beeinträchtigen können;

- krebserregendes Asbest und hochtoxische Quecksilberbestandteile;

- Pestizide mit Substanzen wie Parathion, das beim Anwender bei ungenügendem Schutz gravierende

Vergiftungen auslösen kann. 


\section{$\square$ Internationale Treffen im Umweltbereich}

\section{Globaler Umweltfonds (GEF)}

Der Globale Umweltfonds (GEF) bildet die wichtigste Finanzierungsquelle für umweltbezogene Entwicklungsprojekte von globaler Bedeutung. Der Fonds setzt die Mittel hauptsächlich für den Kampf gegen das Verschwinden der Artenvielfalt, den Klimawandel, die Verschmutzung der internationalen Gewässer und die Zerstörung der Ozonschicht in der Stratosphäre ein. Drei internationale Organisationen - die Weltbank, das Umweltprogramm der Vereinten Nationen (UNEP) sowie das Entwicklungsprogramm der Vereinten Nationen (UNDP) - führen gemeinsam die Aufgaben des GEF durch. Das Portefeuille des GEF umfasst über 500 Projekte in 120 Entwicklungs- oder Schwellenländern. 166 Staaten sind Mitglieder des GEF. Aus dem Fonds wurden seit der Gründung im Jahr 1991 Projekte im Umfang von über zehn Milliarden Franken finanziert. Der Exekutivrat von 32 Mitgliedern legt die Politik des GEF fest und beschliesst die Investitionen. Die Schweiz hat im Exekutivrat einen ständigen Sitz inne.

Eine Delegation der Schweiz unter der Leitung von BUWAL-Direktor Philippe Roch und mit Vertretern des BUWAL, der DEZA und mit dem Exekutivdirektor der Weltbank, einem Schweizer, bereiste vom 21. bis zum 28. Juni 2000 Zentralasien, wo Begegnungen mit den Regierungen von Aserbaidschan, Kirgisistan, Tadschikistan, Turkmenistan und Usbekistan stattfanden. Zweck der Reise war, mit den einzelnen Staaten eine Vereinbarung zu unterzeichnen, um formell eine neue stimmberechtigte Gruppe im Exekutivrat des Globalen Umweltfonds zu schaffen. Diese Gruppe ermöglicht den Partnerländern der Schweiz eine effiziente Vertretung im GEF-Exekutivrat. So sollen Synergien in den Bereichen Umwelt, Handel und Entwicklungszusammenarbeit freigesetzt werden. Einige Staaten der Region wie Kirgisistan gehören bereits zu den Schwerpunktländern der schweizerischen Entwicklungszusammenarbeit. Im Oktober 1999 hat der Bundesrat die Schaffung dieser Gruppe in aller Form gutgeheissen.

[DSDW 1999, Globaler Umweltfonds, S. 144-148.

Internet-Site des Globalen Umweltfonds: www.gefweb.org/

\section{Zwischenstaatliches Forum über Wälder (IFF)- Januar 2000}

Das Zwischenstaatliche Forum über Wälder (Intergovernmental Forum on Forests) wurde von der Kommission für nachhaltige Entwicklung (CSD) eingesetzt, um verschiedene Themen, die anlässlich der Konferenz von Rio im Jahr 1992 aussen vor gelassen wurden, zu vertiefen. Das Forum hielt die vierte und letzte Sitzung vom 31. Januar bis zum 11. Februar 2000 in New York ab. Die Diskussionen bezogen sich in erster Linie auf die umstrittene Frage einer etwaigen Waldkonvention. Die Schweiz befürwortete grundsätzlich die Eröffnung von Verhandlungen zu einer Waldkonvention. Nach mühevollen Diskussionen verständigten sich die Delegierten auf einen Text, der vorsieht, dass der ECOSOC und die Generalversammlung der Vereinten Nationen innerhalb einer Frist von fünf Jahren prüfen sollen, ob es zweckmässig sei, einen Rechtsrahmen für alle Waldarten auszuarbeiten. Die Überlegungen sollen insbesondere in Form des unabhängigen Forums über Wälder, das ein eigenes Sekretariat besitzt, fortgesetzt werden. Im Oktober 2000 gab der Bundesrat seine Zustimmung zur Kandidatur der Schweiz für den Sitz des Waldforums der Vereinten Nationen, das sich in Genf ansiedeln soll. Das neue Organ soll die weltweite Erhaltung und nach- 
haltige Bewirtschaftung der Wälder fördern. Es wird sich vorab mit Fragen der Kontrolle des Handels mit Walderzeugnissen sowie mit der technischen und finanziellen Unterstützung von Entwicklungsländern und der etwaigen Notwendigkeit einer international verbindlichen Waldkonvention befassen. Die Entscheidung über den Sitz dürfte anlässlich der ersten Tagung des Waldforums im Frühjahr 2001 fallen.

$\mathrm{Zu}$ den konkreten Aktionen zum Schutz der Wälder gehört die Forstbescheinigung und das Holzlabel, die darauf abzielen, die Waldbestände unter marktwirtschaftlichen Voraussetzungen zu erhalten. Im Juni 1999 definierten verschiedene Verbände und Organisationen zusammen mit dem BUWAL die Kriterien der Waldzertifizierung für die schweizerische Wirtschaft. Diese «Nationalen Standards für die Waldzertifizierung in der Schweiz » gehen auf die Annäherung zwischen öffentlichen und privaten Interessen in Bezug auf den Wald zurück.

Offizieller Site des Zwischenstaatlichen Forums über Wälder:

www.un.org/esa/sustdev/forests.htm

Earth Negotiation Bulletin: www.iisd.ca/linkages/forestry/forest.html

Site des BUWAL zur Waldpolitik: www.buwal.ch/forst/facts/_.htm

\section{Globales Wasserforum - März 2000}

Das Globale Wasserforum tagte vom 17. bis zum 22. März 2000 in Den Haag. Zur Schweizer Delegation gehörten Vertreter des BUWAL und der DEZA. Anlässlich des Globalen Wasserforums konnte die Schweiz ihre Forderung durchsetzen, dass die 140 Staaten, die Nichtregierungsorganisationen von Weltrang und die Wirtschaftsvertreter öffentlich die Notwendigkeit anerkennen, das Konzept der internationalen Haftpflicht rasch in den Bereich Wasser aufzunehmen, um das Verursacherprinzip dauerhaft zu verwirklichen. Dieses Instrument vorgeschlagen wurde es von der Schweiz (BUWAL) - soll Opfern von grenzüberschreitenden Verschmutzungen nach einem einfachen und direkten Verfahren Schadensersatz gewähren. Ausserdem würde sich eine internationale Regelung der Haftpflicht auch präventiv auswirken, da sich die Unternehmen veranlasst sähen, potenziellen ökologischen Risiken Rechnung zu tragen. Der Erfolg des schweizerischen Vorschlags macht den Weg frei für die Ausarbeitung eines Protokolls über die internationale Haftpflicht im Rahmen der UN-Wirtschaftskommission für Europa (welche Europa, die Vereinigten Staaten und Kanada betrifft).

Die DEZA hat sich ebenfalls an der Konferenz beteiligt und im Jahr 199935 Millionen Franken freigegeben, um das Problem der Trinkwasserversorgung in den Entwicklungsländern zu lösen. Insgesamt erhielten 19 Länder Hilfe von der Schweiz.

Weitere Informationen über das Globale Wasserforum sind auf dem Internet-Site verfügbar: www.unece.org

\section{Kommission für nachhaltige Entwicklung - April/Mai 2000}

Die Kommission für nachhaltige Entwicklung (CSD) wurde als Nachfolgeorgan der Konferenz der Vereinten Nationen für Umwelt und Entwicklung eingesetzt. Sie soll die Umsetzung der verschiedenen Kapitel der Agenda 21 überwachen und vorantreiben. Bei der CSD handelt es sich um eine der neun Fachkommissionen des Wirtschafts- und Sozialrates der Vereinten Nationen. Die CSD versammelte sich wie jedes Jahr im Frühling, vom 24. April bis zum 5. Mai 2000, 
zu ihrer 8. Tagung in New York. Die CSD-8 widmete sich folgenden Schwerpunktthemen: Bodenressourcen, nachhaltige Landwirtschaft und Nutzung der Bodenressourcen, Finanzressourcen, Handel und Investitionen, Wirtschaftswachstum. Ausserdem prüfte die CSD-8 die Schlussfolgerungen und Vorschläge im Schlussbericht des Zwischenstaatlichen Forums über Wälder sowie die Frage der Vorbereitungsarbeiten für Rio +10 . Wie jedes Jahr nahm auch eine Schweizer Delegation an den Arbeiten der Kommission teil.

Earth Negotiation Bulletin, Band über die CSD:

www.iisd.ca/csd/csd8/index.html

Site der Kommission für nachhaltige Entwicklung: www.un.org/esa/sustdev/csd.htm

\section{QUELLEN}

Bundesamt für Umwelt, Wald und Landschaft, Pressemitteilungen 2000. Internet-Site: http://www. buwal.ch/index.htm

United Nations Environment Programme, International environmental Events Calendar. Internet-Site: www.unep.org.

International Institute for Sustainable Development (IISD), Linkages, A multimedia resource for environment and development policy makers. Internet-Site: www.iisd.ca/ 